\title{
Hospital outbreak caused by linezolid resistant Enterococcus faecium in Upper Austria
}

\author{
Heidrun Kerschner ${ }^{1 *+} \mathbb{D}$, Adriana Cabal ${ }^{2,3+}{ }^{2}$, Rainer Hartl ${ }^{1}$, Sigrid Machherndl-Spandl ${ }^{4}$, Franz Allerberger ${ }^{2}$, \\ Werner Ruppitsch ${ }^{2}$ and Petra Apfalter ${ }^{1}$
}

\begin{abstract}
Background: Enterococcus faecium is part of the human gastrointestinal flora but may act as opportunistic pathogen. Environmental persistence, high colonization capability and diverse intrinsic and acquired resistance mechanisms make it especially successful in nosocomial high-risk settings. In March 2014, an outbreak of Linezolid resistant Enterococcus faecium (LREfm) was observed at the hematooncology department of a tertiary care center in Upper Austria.

Methods: We report on the outbreak investigation together with the whole genome sequencing (WGS)-based typing results including also non-outbreak LREfm and susceptible isolates.

Results: The 54 investigated isolates could be divided in six clusters based on cgMLST. Cluster one comprised LREfm isolates of genotype ST117 and CT24, which was identified as the causative clone of the outbreak. In addition, the detection of four other clusters comprising isolates originating from hematooncology patients but also at other hospitals, pointed to LREfm transmission between local healthcare facilities. LREfm patients $(n=36)$ were typically at risk for acquisition of nosocomial pathogens because of immunosuppression, frequent hospitalization and antibiotic therapies. Seven of these 36 patients developed LREfm infection but were successfully treated. After termination of the initial outbreak, sporadic cases occurred despite a bundle of applied outbreak control interventions.

Conclusions: WGS proved to be an effective tool to differentiate several LREfm clusters in an outbreak. Active screening for LREfm is important in a high-risk setting such as hematooncology, where multiple introductions are possible and occur despite intensified infection control measures.
\end{abstract}

Keywords: Enterococcus faecium, Linezolid, Austria, Whole genome sequencing

\section{Background}

Enterococci are gram-positive bacteria found in the environment and as part of the human gastrointestinal flora [1]. They can act as opportunistic pathogens causing a broad range of diseases such as blood stream or wound-associated infections [2]. Hospital-adapted clones such as clonal complex (CC)17 Enterococcus faecium show persistence in the environment and high colonization capability [3]. E. faecium has diverse intrinsic resistance mechanisms to antibiotics

\footnotetext{
* Correspondence: heidrun.kerschner@analyse.eu

${ }^{\dagger}$ Heidrun Kerschner and Adriana Cabal contributed equally to this work.

${ }^{1}$ National Reference Center for Antimicrobial Resistance and Nosocomial Infections, Institute for Hygiene, Microbiology and Tropical Medicine, Ordensklinikum Linz Elisabethinen, Fadingerstrasse 1, 4020 Linz, Austria Full list of author information is available at the end of the article
}

and is able to progressively acquire antimicrobial resistances such as to ampicillin and vancomycin (VAN), thus limiting the therapeutic options [4]. One therapy of last resort against vancomycin-resistant Enterococci (VRE) is the oxazolidinone linezolid (LZD), which inhibits protein synthesis by binding to the 50S 23S rRNA [1]. Resistance to LZD has already been reported in E. faecium with the most common resistance mechanisms referring to mutations in the $\mathrm{V}$ domain of the $23 \mathrm{~S}$ rRNA [5, 6]. Mutations in the sequence of genes encoding the riboproteins L3, L4 and L22 account for the second most common mechanisms. Third, recently described plasmid-mediated resistances due to $c f r$ [7], optrA [8] and poxtA [9] and finally, yet unknown LZD resistance mechanisms are known to exist [10]. Risk factors associated with LZD resistance

(c) The Author(s). 2019 Open Access This article is distributed under the terms of the Creative Commons Attribution 4.0 International License (http://creativecommons.org/licenses/by/4.0/), which permits unrestricted use, distribution, and reproduction in any medium, provided you give appropriate credit to the original author(s) and the source, provide a link to the Creative Commons license, and indicate if changes were made. The Creative Commons Public Domain Dedication waiver (http://creativecommons.org/publicdomain/zero/1.0/) applies to the data made available in this article, unless otherwise stated. 
include transplants and surgery, immunosuppression and previous or ongoing treatment with LZD [11, 12]. Usually, LZD resistant E. faecium (LREfm) strains emerge in patients 22125 days after treatment [13]. LREfm outbreaks tend to be mostly colonizations, although clinical outbreaks with invasive LREfm infections have been reported [14].

\section{Methods}

In March 2014, routine surveillance cultures of stool and urine from four patients hospitalized at the department of internal medicine 1 (DIM1) of an Austrian tertiary care center tested positive for LREfm. An outbreak investigation was then initiated to identify the source of the outbreak, characterize the outbreak strain by whole genome sequencing (WGS) and antimicrobial susceptibility testing and apply control measures.

\section{Hospital and ward description}

The DIM1 is one of three departments of internal medicine at a tertiary care center (hospital 4) in Linz, Upper Austria. It consists of a general oncology ward (16 rooms for 33 patients), a leukemia and autologous stem cell transplantation ward (8 rooms for 12 patients), a stem cell transplantation unit ( 5 rooms for 5 patients) and an outpatient clinic. The bed occupancy rate ranges between 90 and 100\%.

\section{Outbreak description}

Between March and May 2014, we identified ten LREfm colonized patients and one patient with LREfm bloodstream infection who were all hospitalized at the DIM1. The outbreak was contained in June 2014 through implementation of control measures. Active case finding detected twelve additional patients at the DIM1 and four at other units of hospital 4 till the end of the year 2017 (total number of cases: 27). Thirteen of these 16 patients were categorized as colonized, one patient treated at DIM1 had LREfm bloodstream infection and two patients treated in other departments had a urinary tract infection and a surgical site infection, respectively. A case was defined as any patient with culture-confirmed LREfm identified at hospital 4 from the beginning March 2014 onwards. Cases were included in this study until the end of 2017.

\section{Patients}

In total, 36 patients were included in the study. Twentyseven of those were the cases from hospital 4 as described above (24 were patients of the DIM1 and three were patients from other wards), who were designated as patient collective A. In order to gain insight into the occurrence of the outbreak strain in the local and Austrian hospital community, an additional nine patients belonging to six other hospitals (hospitals 1 to 3 and 5 to 7 ) in the provinces of Carinthia, Upper Austria and Vienna were included in the study and denominated patient collective B. Their isolates had been collected between 2012 and 2018. Demographic and epidemiological data such as patient outcome (death/survival), immune status, routine screening or clinical sampling, hospital contact in the previous year and other parameters were collected, anonymized and then analyzed using Microsoft Excel 2016 and SPSS statistical software version 22.0 (Chicago, Illinois). Data on the exposure to LZD in the 28 days prior to isolation of LREfm and current antibiotic treatment (yes/no) was retrieved from patient records at the time of the first LREfm isolation. If available, duration of LREfm carriage was estimated from surveillance culture data. LZD consumption data of the DIM1 were extracted from the hospital pharmacy database and analyzed by AVS.webKess software [15]. We investigated at patient level (patient collectives A and $\mathrm{B}, n=36)$ the acquisition of the outbreak strain and possible associations with their demographics and epidemiological characteristics using STATA 13 software.

\section{Isolates}

Isolates $(n=54)$ were retrieved from cryobanks (Mast, Reinfeld, Germany) and cultured on blood agar (Oxoid, Wesel, Germany). Maldi-TOF analysis (Bruker Daltonics, Bremen, Germany) was used for species confirmation. LZD susceptibility testing was done using disk diffusion and, for the 45 LREfm isolates, gradient testing (Biomérieux, Marcy L'Étoile, France) according to EUCAST criteria and breakpoints (resistant: $>4 \mathrm{mg} / \mathrm{L}$ or $<19 \mathrm{~mm}$, respectively). Additionally, Tedizolid (TDZ) gradient testing was performed for all LREfm (Liofilchem, Roseto degli Abruzzi, Italy) according to the manufacturer's instructions. VAN susceptibility data were retrieved from the routine antibiogram. Finally, all LREfm were investigated for the presence of optrA and cfr using previously published primer sets [16].

For comparison of WGS-based typing data, we included nine additional LZD susceptible E. faecium (LSEfm) isolates (eight from blood culture and one from stool) recovered from eight patients staying at hospitals 4 and 6 between May 2014 and June 2017 (patient collective C). Two of those were collected from patients having also an LREfm isolate. The others were chosen because they were invasive isolates from affected wards.

\section{DNA extraction, WGS and typing}

High-molecular-weight DNA from the 54 bacterial overnight cultures was isolated using a MagAttract HMW DNA kit (Qiagen, Hilden, Germany). DNA was quantified using DropSense 16 (Trinean NV/SA, Gentbrugge, Belgium). Library for WGS was prepared with a NexteraXT kit (Illumina, Inc., San Diego, CA, USA) according to manufacturer's instructions and a 300-bp paired-end sequencing run was performed on an Illumina MiSeq instrument using the MiSeq 
V3 reagent kit (Illumina Inc., San Diego, CA, USA) for the 54 E. faecium isolates. Raw reads were de novo assembled into draft genomes using SPAdes version 3.11.1 [17]. Contigs were then filtered for a minimum coverage of 5 and minimum length of 200 base pairs. SeqSphere+ software (Ridom $\mathrm{GmbH}$, Münster, Germany) was used for strain typing using a public core genome multilocus sequence typing (cgMLST) scheme [18]. Minimum spanning trees (MST) were generated to illustrate the number of allelic differences between isolates and visualize clusters. The allelic cluster threshold was set to $\leq 20$ allelic differences as previously proposed [18]. Sequence types (STs) from the classical MLST [19] were in silico extracted from WGS data using SeqSphere+. Likewise, the three genes encoding the riboproteins L3, L4 and L22 and the 23S rRNA genes were extracted and screened for point mutations associated to LZD resistance by comparing their sequences with those of the E. faecium DO reference strain Antimicrobial resistance genes including those conferring LZD resistance (optrA, poxtA and cfr and its variants) were identified via the Comprehensive Antibiotic Resistance Database (CARD) [20]. In addition, both the point mutations and the genes conferring LZD resistance including G2576 U located in the 23S rRNA were double-checked using LRE finder [21]. The presence of specific virulence genes (VGs) for E. faecium among the isolates was investigated using the Virulence Factors Database and Virulence Finder 2.0 [22, 23]. Plasmid Finder was used for plasmid identification among the sequenced isolates [24]. Lastly, we assessed at an isolate level $(n=54)$ possible associations between the resistance phenotype (LREfm/LSEfm) of the isolates and the presence of VGs, resistance genes and the point mutation 23S rRNA G2576U.

\section{Results}

\section{Characteristics of all study patients and epidemiological data}

In the study period a total of LREfm 36 patients were detected (patient collectives A and B). Their demographic and epidemiological characteristics are summarized in Table 1. Figure 1a shows the epidemiological curve of the outbreak by collection date of the first LREfm isolate. Patients with more than one isolate are represented once only in the epicurve. The location of the hospitals is shown in Fig. 1b.

\section{Patient movement and clinical characteristics}

When patient movement of cases (collective A) was traced, possible direct transmission could be shown for 10 out of 11 cases during the initial outbreak event (March 2014 to May 2014). Five of the eleven cases shared a room with confirmed LREfm cases and another five were treated in wards at the same time as confirmed cases (Fig. 2).

Of the 16 additional cases detected between September 2014 and November 2017, six shared at least the DIM1
Table 1 Clinical and epidemiological data obtained for the 36 patients of collective $A$ and $B$

\begin{tabular}{|c|c|c|}
\hline \multicolumn{3}{|l|}{ Characteristics } \\
\hline Age, years, median (range) & $57(22-91)$ & \\
\hline \multicolumn{3}{|l|}{ Sex, n (\%) } \\
\hline Male & 23 & 63.9 \\
\hline Female & 13 & 36.1 \\
\hline \multicolumn{3}{|l|}{ Patient status, n (\%) } \\
\hline Inpatient & 35 & 97.2 \\
\hline Outpatient & 1 & 2.8 \\
\hline \multicolumn{3}{|l|}{ Transfer from other Hospital/LTCF, n (\%) } \\
\hline Yes & 8 & 22.2 \\
\hline No & 25 & 69.4 \\
\hline Unknown & 3 & 8.3 \\
\hline \multicolumn{3}{|l|}{ LZD therapy (previous 28 days), n (\%) } \\
\hline Yes & 10 & 27.8 \\
\hline No & 25 & 69.4 \\
\hline Unknown & 1 & 2.8 \\
\hline Duration of LZD therapy, days, median (range) & $9(3-35)$ & \\
\hline \multicolumn{3}{|l|}{ LREfm status, n (\%) } \\
\hline Infection & 4 & 11.1 \\
\hline Colonization & 30 & 83.3 \\
\hline Unknown & 2 & 5.6 \\
\hline Duration of colonization, days, median (range) & $22(3-245)$ & \\
\hline \multicolumn{3}{|l|}{ Current antibiotic therapy, n (\%) } \\
\hline Yes & 36 & 100.0 \\
\hline No & 0 & 0.0 \\
\hline \multicolumn{3}{|l|}{ Hospital contact in previous year, $\mathrm{n}(\%)$} \\
\hline Yes & 31 & 86.1 \\
\hline No & 2 & 5.6 \\
\hline Unknown & 3 & 8.3 \\
\hline \multicolumn{3}{|l|}{ Underlying disease, $\mathrm{n}(\%)$} \\
\hline Hematology-Oncology & 25 & 69.4 \\
\hline allogeneic SCT & 17 & \\
\hline autologous SCT & 3 & \\
\hline Trauma/orthopedic & 4 & 11.1 \\
\hline Abdominal surgery/Pancreatitis & 4 & 11.1 \\
\hline Other & 3 & 8.3 \\
\hline \multicolumn{3}{|l|}{ Immunocompromised, n (\%) } \\
\hline Yes & 31 & 86.1 \\
\hline No & 4 & 11.1 \\
\hline Unknown & 1 & 2.8 \\
\hline \multicolumn{3}{|l|}{ Died during follow-up (until end of 2017), n (\%) } \\
\hline Yes & 15 & 41.7 \\
\hline No & 14 & 38.9 \\
\hline Unknown & 7 & 19.4 \\
\hline
\end{tabular}


Table 1 Clinical and epidemiological data obtained for the 36 patients of collective A and B (Continued)

\begin{tabular}{lll}
\hline Characteristics & & \\
\hline Source material first LREfm isolate, $\mathrm{n}(\%)$ & 18 & 50.0 \\
Stool & 9 & 25.0 \\
Urine & 7 & 19.4 \\
Swab (wound, throat, eye) & 1 & 2.8 \\
Blood culture & 1 & 2.8 \\
Catheter tip & & \\
Source material secondary isolates, $\mathrm{n}(\%)$ & 6 & \\
Stool & 2 & \\
Blood culture & 1 & \\
Urine & & 66.6 \\
Screening sample, $\mathrm{n}(\%)$ & 24 & 30.5 \\
Yes & 11 & 2.77 \\
No & 1 & \\
Unknown &
\end{tabular}

with known LREfm carriers during their hospitalization. For ten cases, no direct epidemiological link could be established except for possible indirect transmission on previously affected wards.

Most patients (collective A + B) had multiple LREfm isolates, typically from stool and urine, but eventually lost those enterococci again. To estimate duration of colonization, time from first LREfm isolate until clearance was available for 21 patients and ranged from three to 245 days (median 22 days). For only two patients the same LREfm clone could be cultured again after more than 1 year.

Five patients staying at hospital 4, one at hospital 6 and one at hospital 1 had an LREfm infection (total $n=$ 7 ), the rest was considered colonized. All seven infected patients were treated with VAN and none of them died. Fifteen $(41.6 \%)$ patients died during follow-up but only two died during their stay at hospital 4 at the time of LREfm detection of unrelated causes. All patients (100\%) received antibiotics at the time of LREfm detection. Ten

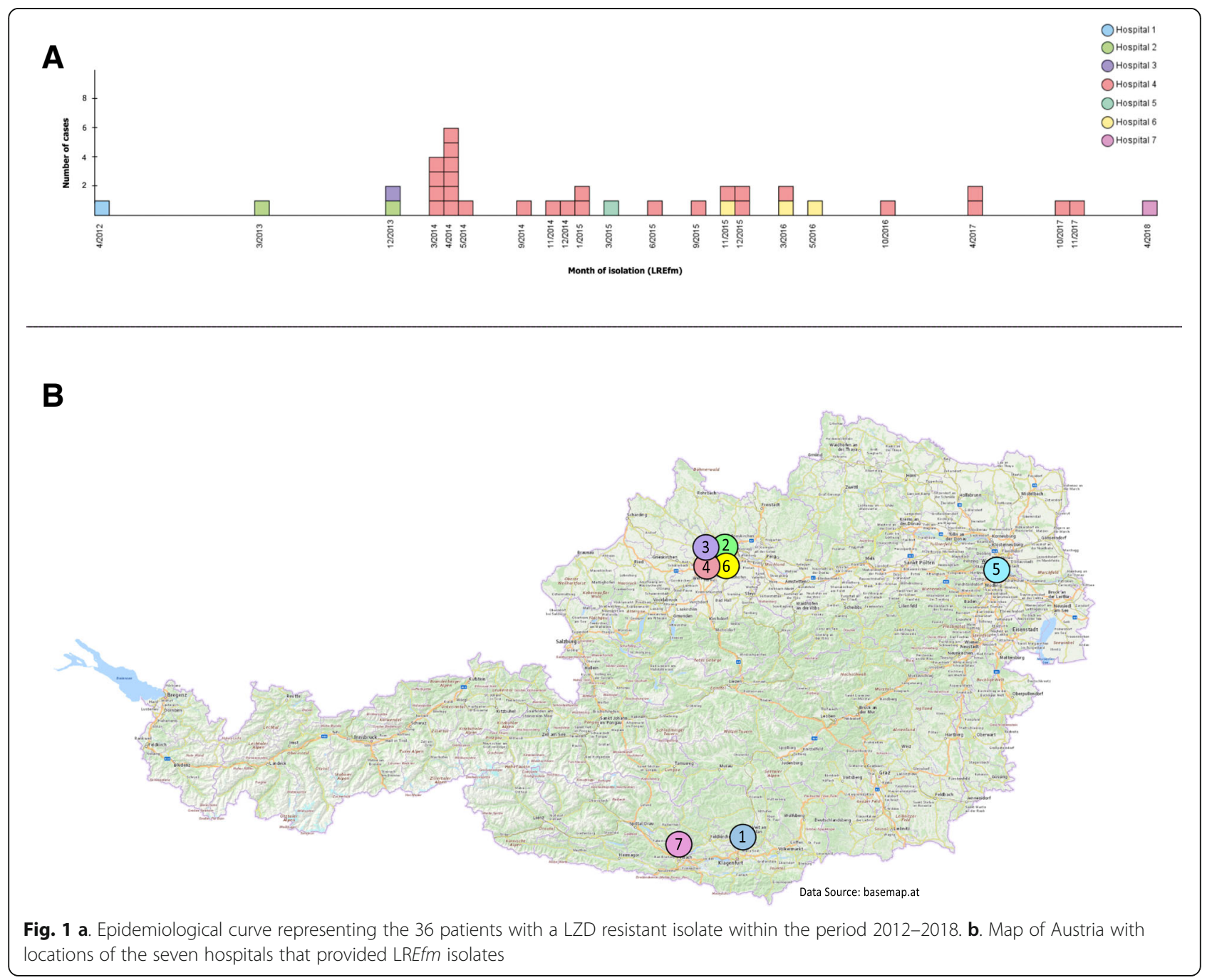




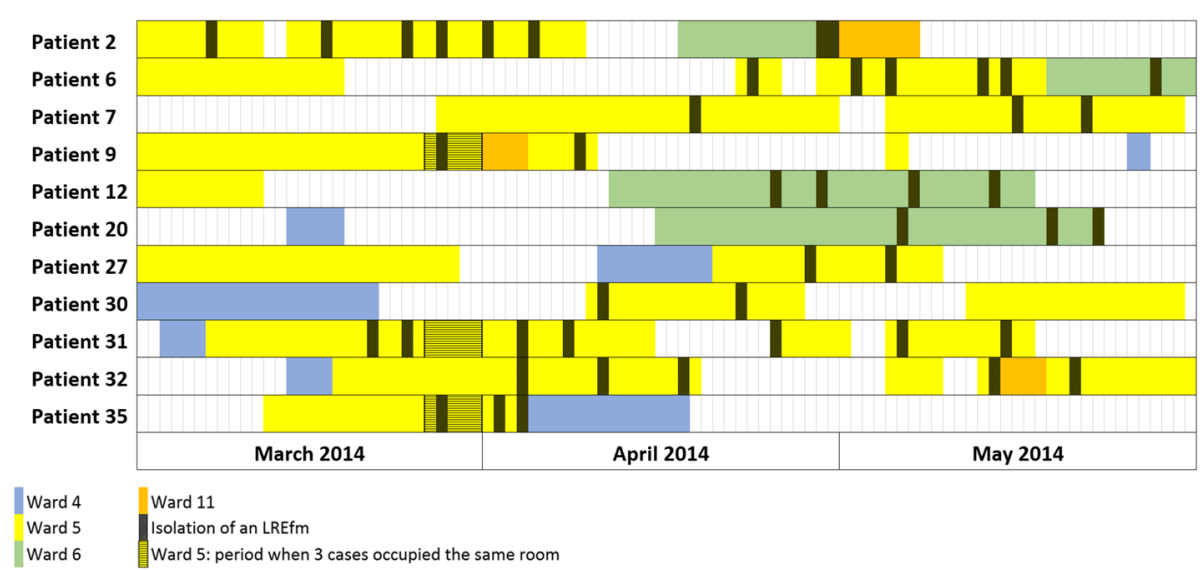

Fig. 2 Patient trace at hospital 4 for the months March to June 2014. Each box corresponds to one day. The striped area marks the time when three of the first four cases occupied the same room on ward 5

(27.8\%) patients had received LZD in the 28 days prior to the recovery of LREfm, whereas 25 had not been exposed. Eight patients (22.2\%), all from hospital 4, had been transferred from other hospitals, two of them from hospital 6. Thirty-one (81.1\%) patients had been hospitalized in the previous year and the same number (81.1\%) were immunocompromised. The majority of patients $(n=24,66.6 \%)$ had been tested for LREfm as part of screening.

\section{Antimicrobial susceptibility testing}

The LREfm isolates were all LZD resistant by disk diffusion testing and had a median MIC of $32 \mathrm{mg} / \mathrm{L}$ (range 4 to > 256). The two isolates with an MIC of $4 \mathrm{mg} / \mathrm{L}$ were included because of their LZD zone diameters of 17 and $18 \mathrm{~mm}$, respectively. TZD MICs ranged from 1 to 32 $\mathrm{mg} / \mathrm{l}$ (median $2 \mathrm{mg} / \mathrm{L}$ ) and were all lower than the respective LZD MICs. Three isolates showed MICs of > $256 \mathrm{mg} / \mathrm{l}$ for VAN.

\section{WGS-based typing}

WGS-based typing assigned the 54 isolates to seven sequence types (STs) (Table 2), with ST117 ( $n=30 ; 55.5 \%)$ and ST80 $(n=18 ; 33.3 \%)$ being the most frequent. Within the LREfm group $(n=45)$, ST117 was the predominant ST $(n=28,62 \%)$ while for the LSEfm ST80 was the most frequent ST $(n=5,56 \%)$. The vast majority of ST117 isolates belonged to cluster type (CT) 24 ( $n=$ $25,83 \%)$. All the other STs were found once or twice. Based on the cgMLST data of the 54 isolates, the MST revealed six clusters (Fig. 3). We only identified one cluster (cluster 1) with 25 LREfm isolates (46.3\%, from 20 patients overall) as part of the outbreak at the DIM1 in hospital 4. An LSEfm isolate from a case also clustered in cluster 1 . All isolates within the cluster were
ST117 and all but one presented CT24. Isolates of cluster 1 differed by 0 to 10 alleles.

WGS-based typing revealed that 10 out of 11 cases (see also Fig. 2, all patients except patient 30 / Ef-13) detected at the beginning of the outbreak were part of it and therefore their isolates $(n=15)$ belonged to cluster 1 , confirming the transmission suspected by patient movement data. After May 2014, nine cases were detected and these also carried the outbreak clone $(n=10$ isolates, grouping in cluster 1). Last, seven additional cases from hospital 4 detected after May 2014 were not part of the outbreak, grouping five LREfm isolates in clusters $2(n=2)$ and 3 $(n=3)$. At a patient level $(n=36)$, we found significant associations between acquisition of the LREfm outbreak strain and screening sampling (Exact $p<0.005$ ) as well as a fatal outcome during follow-up (Exact $p<0.005$ ).

Clusters 2 to 6 comprised isolates from patients staying at different hospitals and from a wider period (2012-2018) (Fig. 3). Cluster 2 (ST80, CT1873) comprised two LREfm isolates from two patients of hospital 4 , not treated at the DIM1, four LREfm isolates from four patients at hospitals $2(n=2), 3(n=1)$ and $6(n=1)$ and also one LSEfm isolate from hospital 6 . None of these patients was treated for an oncological disease, they were typically older (median age 69 years) and two had been transferred from long term care facilities.

Cluster 3 (ST80, CT16) consisted of five isolates from four patients treated at the DIM1, of which two were LSEfm. Cluster 4 (ST80, CT315) grouped three isolates from three patients staying at hospitals $6(n=2)$ and 4 $(\mathrm{n}=1)$, the latter being an LSEfm from a patient who had not been treated at the DIM1. Cluster 5 (ST203, CT20) grouped two isolates from a patient staying at hospital 5 in Vienna. Cluster 6 (ST117, CT929) comprised two isolates obtained at two hospitals located in different regions. One of them originated from hospital 
Table 2 Characteristics of the 54 E. faecium isolates based on patient data and molecular results

\begin{tabular}{|c|c|c|c|c|c|c|c|c|c|c|c|c|c|}
\hline Case & Province & Hospital & Ward & Isolate & $\begin{array}{l}\text { Isolation } \\
\text { year }\end{array}$ & Source & Phenotype & $\begin{array}{l}\text { LRE } \\
\text { mechanism }\end{array}$ & ST & CT & $\begin{array}{l}\text { Cluster } \\
\text { number }^{\mathrm{a}}\end{array}$ & $\begin{array}{l}\text { Linezolid MIC } \\
\text { (mg/L) }\end{array}$ & $\begin{array}{l}\text { Tedizolid MIC } \\
\text { (mg/L) }\end{array}$ \\
\hline 1 & UA & 2 & 2 & Ef-03 & 2013 & $\begin{array}{l}\text { Wound } \\
\text { swab }\end{array}$ & LREfm & unknown & 80 & 1873 & 2 & 32 & 4 \\
\hline 2 & UA & 4 & 5 & Ef-11 & 2014 & Stool & LREfm & $\begin{array}{l}\text { G2576U, } \\
\text { A2598G }\end{array}$ & 117 & 24 & 1 & $>256$ & 32 \\
\hline 2 & UA & 4 & 6 & Ef-22 & 2014 & $\begin{array}{l}\text { Blood } \\
\text { culture }\end{array}$ & LREfm & unknown & 117 & 24 & 1 & 32 & 4 \\
\hline 3 & UA & 6 & 13 & Ef-44 & 2016 & $\begin{array}{l}\text { Wound } \\
\text { swab }\end{array}$ & LREfm & unknown & 80 & 1873 & 2 & 64 & 2 \\
\hline 4 & UA & 4 & 10 & Ef-36 & 2015 & $\begin{array}{l}\text { Wound } \\
\text { swab }\end{array}$ & LREfm & unknown & 80 & 1873 & 2 & 128 & 4 \\
\hline 6 & UA & 4 & 5 & Ef-17 & 2014 & Urine & LREfm & G2576U & 117 & 24 & 1 & 32 & 8 \\
\hline 6 & UA & 4 & 5 & Ef-20 & 2014 & Stool & LREfm & unknown & 117 & 24 & 1 & 64 & 2 \\
\hline 7 & UA & 4 & 5 & Ef-15 & 2014 & Urine & LREfm & unknown & 117 & 24 & 1 & 16 & 4 \\
\hline 8 & UA & 4 & 11 & Ef-43 & 2016 & Throat & LREfm & G2576U & 117 & 24 & 1 & 32 & 2 \\
\hline 9 & UA & 4 & 5 & Ef-05 & 2014 & Stool & LREfm & unknown & 117 & 24 & 1 & 16 & 2 \\
\hline 10 & V & 5 & N/A & Ef-24 & 2015 & Stool & LREfm & unknown & 203 & 20 & 5 & 16 & 2 \\
\hline 10 & V & 5 & $\mathrm{~N} / \mathrm{A}$ & Ef-25 & 2015 & Stool & LREfm & unknown & 203 & 20 & 5 & 16 & 2 \\
\hline 11 & UA & 4 & 5 & Ef-40 & 2015 & Stool & LREfm & unknown & 117 & 24 & 1 & 32 & 2 \\
\hline 12 & UA & 4 & 6 & Ef-18 & 2014 & Urine & LREfm & G2576U & 117 & 24 & 1 & 32 & 2 \\
\hline 13 & UA & 4 & 5 & Ef-37 & 2015 & Stool & LREfm & unknown & 117 & 24 & 1 & 64 & 2 \\
\hline 14 & UA & 4 & 6 & 13639 & 2015 & $\begin{array}{l}\text { Blood } \\
\text { culture }\end{array}$ & LSEfm & unknown & 117 & 24 & 1 & n.d. & n.d. \\
\hline 14 & UA & 4 & 6 & Ef-31 & 2015 & Stool & LREfm & unknown & 117 & 24 & 1 & 32 & 2 \\
\hline 15 & UA & 4 & 9 & Ef-30 & 2014 & Urine & LREfm & unknown & 80 & 1873 & 2 & 64 & 4 \\
\hline 16 & UA & 4 & 5 & Ef-28 & 2014 & Urine & LREfm & G2576U & 117 & 24 & 1 & 64 & 8 \\
\hline 17 & UA & 4 & 6 & Ef-35 & 2015 & Stool & LREfm & unknown & 80 & 16 & 3 & 64 & 4 \\
\hline 19 & UA & 4 & 7 & Ef-26 & 2017 & Urine & LREfm & G2576U & 117 & 1875 & $\begin{array}{l}\text { not } \\
\text { clustered }\end{array}$ & 64 & 8 \\
\hline 20 & UA & 4 & 6 & Ef-23 & 2014 & Stool & LREfm & $\begin{array}{l}\text { G2576U, } \\
\text { A2598G }\end{array}$ & 117 & 1872 & 1 & 32 & 4 \\
\hline 20 & UA & 4 & 6 & Ef-27 & 2014 & Stool & LREfm & G2576U & 117 & 24 & 1 & 32 & 16 \\
\hline 21 & UA & 6 & 12 & Ef-42 & 2016 & Catheter & LREfm & $\begin{array}{l}\text { G2576U, } \\
\text { A2598G }\end{array}$ & 80 & 315 & 4 & 64 & 4 \\
\hline 22 & UA & 2 & 1 & Ef-02 & 2013 & $\begin{array}{l}\text { Wound } \\
\text { swab }\end{array}$ & LREfm & G2576U & 80 & 1873 & 2 & 128 & 16 \\
\hline 23 & UA & 4 & 5 & Ef-39 & 2015 & Stool & LREfm & unknown & 117 & 24 & 1 & 4 & 1 \\
\hline 24 & UA & 4 & 6 & Ef-29 & 2014 & Stool & LREfm & unknown & 117 & 24 & 1 & 8 & 2 \\
\hline 25 & UA & 4 & 5 & Ef-48 & 2017 & Stool & LREfm & unknown & 117 & 24 & 1 & 16 & 1 \\
\hline 26 & UA & 4 & 5 & Ef-46 & 2017 & Stool & LREfm & unknown & 117 & 24 & 1 & 32 & 1 \\
\hline 27 & UA & 4 & 5 & Ef-19 & 2014 & Urine & LREfm & unknown & 117 & 24 & 1 & 32 & 2 \\
\hline 27 & UA & 4 & 5 & Ef-21 & 2014 & Stool & LREfm & unknown & 80 & 1879 & $\begin{array}{l}\text { not } \\
\text { clustered }\end{array}$ & 4 & 1 \\
\hline 28 & UA & 4 & 5 & Ef-47 & 2017 & Stool & LREfm & unknown & 80 & 1876 & $\begin{array}{l}\text { not } \\
\text { clustered }\end{array}$ & 32 & 2 \\
\hline 29 & UA & 4 & 6 & 14921 & 2016 & $\begin{array}{l}\text { Blood } \\
\text { culture }\end{array}$ & LSEfm & unknown & 80 & 16 & 3 & n.d. & n.d. \\
\hline 29 & UA & 4 & 5 & Ef-32 & 2015 & Stool & LREfm & unknown & 80 & 16 & 3 & 64 & 2 \\
\hline
\end{tabular}


Table 2 Characteristics of the 54 E. faecium isolates based on patient data and molecular results (Continued)

\begin{tabular}{|c|c|c|c|c|c|c|c|c|c|c|c|c|c|}
\hline Case & Province & Hospital & Ward & Isolate & $\begin{array}{l}\text { Isolation } \\
\text { year }\end{array}$ & Source & Phenotype & $\begin{array}{l}\text { LRE } \\
\text { mechanism }\end{array}$ & ST & CT & $\begin{array}{l}\text { Cluster } \\
\text { number }^{a}\end{array}$ & $\begin{array}{l}\text { Linezolid MIC } \\
\text { (mg/L) }\end{array}$ & $\begin{array}{l}\text { Tedizolid MIC } \\
\text { (mg/L) }\end{array}$ \\
\hline 29 & UA & 4 & 5 & Ef-33 & 2015 & $\begin{array}{l}\text { Blood } \\
\text { culture }\end{array}$ & LREfm & unknown & 80 & 16 & 3 & 64 & 4 \\
\hline 29 & UA & 4 & 5 & $E f-41$ & 2016 & Stool & LSEfm & unknown & 117 & 929 & 6 & 2 & 2 \\
\hline 30 & UA & 4 & 5 & Ef-13 & 2014 & Stool & LREfm & unknown & 117 & 1878 & $\begin{array}{l}\text { not } \\
\text { clustered }\end{array}$ & 16 & 2 \\
\hline 30 & UA & 4 & 5 & Ef-14 & 2014 & Stool & LREfm & G2576U & 117 & 24 & 1 & 8 & 2 \\
\hline 30 & UA & 4 & 6 & Ef-34 & 2015 & Urine & LREfm & unknown & 117 & 24 & 1 & 64 & 2 \\
\hline 31 & UA & 4 & 5 & Ef-07 & 2014 & Urine & LREfm & unknown & 117 & 24 & 1 & 16 & 2 \\
\hline 31 & UA & 4 & 5 & Ef-08 & 2014 & Stool & LREfm & unknown & 117 & 24 & 1 & 16 & 2 \\
\hline 32 & UA & 4 & 5 & Ef-12 & 2014 & Stool & LREfm & unknown & 117 & 24 & 1 & 16 & 2 \\
\hline 33 & UA & 3 & 3 & Ef-04 & 2013 & $\begin{array}{l}\text { Eye } \\
\text { swab }\end{array}$ & LREfm & G2576U & 80 & 1873 & 2 & 32 & 4 \\
\hline 34 & UA & 6 & 16 & Ef-38 & 2015 & $\begin{array}{l}\text { Blood } \\
\text { culture }\end{array}$ & LREfm & unknown & 80 & 315 & 4 & 32 & 2 \\
\hline 35 & UA & 4 & 5 & Ef-09 & 2014 & Stool & LREfm & G2576U & 117 & 24 & 1 & 16 & 2 \\
\hline 36 & C & 1 & 1 & Ef-01 & 2012 & $\begin{array}{l}\text { Wound } \\
\text { swab }\end{array}$ & LREfm & unknown & 192 & 1877 & $\begin{array}{l}\text { not } \\
\text { clustered }\end{array}$ & 16 & 2 \\
\hline 37 & UA & 4 & 5 & Ef-45 & 2016 & Stool & LREfm & unknown & 78 & 1874 & $\begin{array}{l}\text { not } \\
\text { clustered }\end{array}$ & 32 & 4 \\
\hline 38 & UA & 6 & 15 & 14962 & 2016 & $\begin{array}{l}\text { Blood } \\
\text { culture }\end{array}$ & LSEfm & n.a. & 1479 & $\begin{array}{l}\text { not } \\
\text { assigned }\end{array}$ & $\begin{array}{l}\text { not } \\
\text { clustered }\end{array}$ & n.d. & n.d. \\
\hline 39 & UA & 4 & 5 & 12981 & 2014 & $\begin{array}{l}\text { Blood } \\
\text { culture }\end{array}$ & LSEfm & n.a. & 80 & 16 & 3 & n.d. & n.d. \\
\hline 40 & C & 7 & 14 & Ef-51 & 2018 & Urine & LREfm & G2576U & 117 & 929 & 6 & 16 & 2 \\
\hline 41 & UA & 6 & 15 & 14522 & 2015 & $\begin{array}{l}\text { Blood } \\
\text { culture }\end{array}$ & LSEfm & n.a. & 80 & 1873 & 2 & n.d. & n.d. \\
\hline 42 & UA & 4 & 4 & 15549 & 2016 & $\begin{array}{l}\text { Blood } \\
\text { culture }\end{array}$ & LSEfm & n.a. & 1466 & $\begin{array}{l}\text { not } \\
\text { assigned }\end{array}$ & $\begin{array}{l}\text { not } \\
\text { clustered }\end{array}$ & n.d. & n.d. \\
\hline 43 & UA & 4 & 7 & 15378 & 2016 & $\begin{array}{l}\text { Blood } \\
\text { culture }\end{array}$ & LSEfm & n.a. & 80 & $\begin{array}{l}\text { not } \\
\text { assigned }\end{array}$ & 4 & n.d. & n.d. \\
\hline 45 & UA & 4 & 4 & 15925 & 2017 & $\begin{array}{l}\text { Blood } \\
\text { culture }\end{array}$ & LSEfm & n.a. & 80 & 467 & $\begin{array}{l}\text { not } \\
\text { clustered }\end{array}$ & n.d. & n.d. \\
\hline
\end{tabular}

${ }^{a}$ Numbering allocated for each cluster in the MST

n.a. not applicable, n.d. not done

C Carinthia, CT Cluster type (cgMLST), MST minimum spanning tree, ST Sequence Type (classical MLST), UA Upper Austria, V Vienna

7 in Carinthia and the other one was an LSEfm stool isolate from a DIM1 patient at hospital 4, from whom also three LREfm isolates were included in the study all clustering in cluster 3 . The timespan between obtainment of cluster 6 isolates was 2 years and there was no epidemiological data to explain their relatedness.

Last, a number of isolates did not cluster with any other isolate: five LREfm from five patients at hospital 4, three LSEfm from two patients at hospital 4 and one at hospital 6 and one isolate from one patient at hospital 1.

Twenty-four resistance genes were detected among the 54 isolates (Additional file 1: Table S1). The most common ones were AAC $\left(6^{\prime}\right)$-li $(n=52 ; 96.3 \%)$, efmA $(\mathrm{n}=52$; $96.3 \%)$ and ermB ( $n=48 ; 88.9 \%)$. Moreover, the multidrug efflux pump efmA was detected among all LREfm isolates within cluster 1 . Significantly higher proportions were found for $d f r F$ (Fisher's exact $P=0.0020$ ) and sat4 (Fisher's exact $P=0.0499)$ within the LREfm $(n=45)$.

PCRs targeting $c f r$ and optrA genes were negative for all LREfm. These genes, as well as poxtA, were also absent when blasting WGS data against the CARD database, the LRE tool from CGE server and Plasmid finder. Thirteen out of $36(36.1 \%)$ cases presented at least one LREfm isolate carrying the point mutation G2576U at the $23 \mathrm{~S}$ rRNA, as revealed when using CARD database meaning that $25.9 \%(n=14)$ of the E. faecium isolates carried that point mutation (Table 2 and Additional file 1: Table S1). The number of $23 \mathrm{~S}$ rRNA mutated copies varied between 2 and 6 . In addition, three of those isolates carried a novel point mutation at A2598G of the 


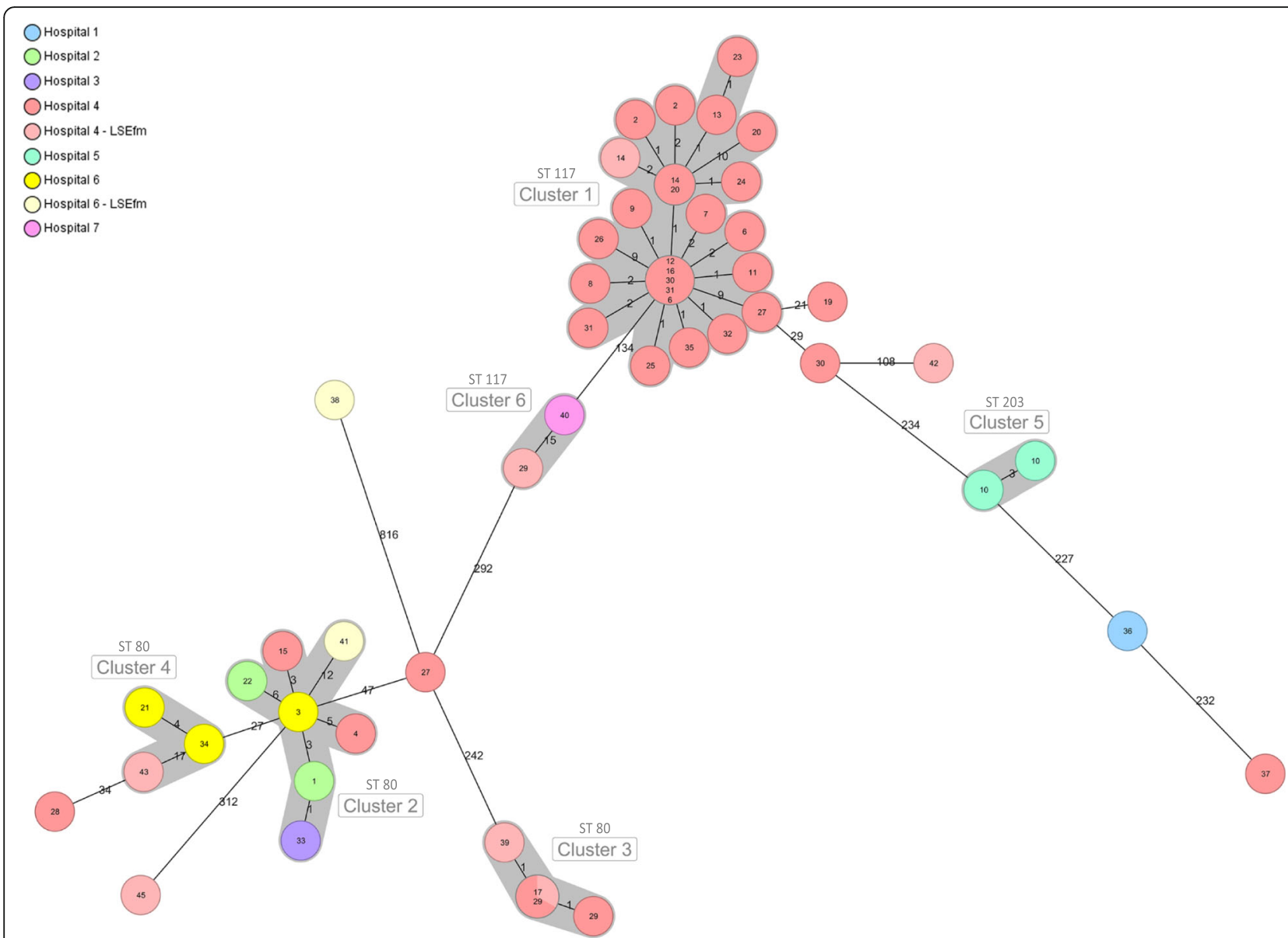

Fig. 3 Minimum spanning tree showing the genetic relationship among the 54 sequenced isolates based on cgMLST. Each circle represents one or more isolates, color-coded by hospital of origin. Numbers inside each circle represent the patient ID. Shaded areas depict clusters. Connection lines indicate the number of allelic differences between the isolates. All isolates are LREfm except those ones that are light-colored, which represent LSEFm isolates

$23 \mathrm{~S}$ rRNA. For 31 LREfm isolates, the resistance mechanism was not found.

The three VAN resistant isolates (the two ST203 isolates from cluster 5 and a non-clustered ST117 isolate) carried the gene vanA.

Ten VGs were detected among the typed LREfm and LSEfm isolates (Additional file 1: Table S2). The most frequently found VGs were $\operatorname{sgrA}(n=53,98 \%), \operatorname{acm}(n=47$, $87 \%)$ and $e c b \mathrm{~A}(n=33,61.1 \%)$ and only ecbA was found to be significantly more frequent (Fisher's exact $P=0.013$ ) among LREfm isolates. We did not detect associations between ST and any of the VGs tested.

\section{Outbreak control measures}

After the detection of the outbreak, active surveillance was put in place for the DIM1 including antimicrobial susceptibility tests for all clinical and screening $E$. faecium isolates. In addition, strict contact precautions for known colonized patients, single-room patient care and increased frequency of cleaning and disinfection of patient-near-surfaces especially in bathrooms was initiated. Moreover, LZD consumption was reduced by preferential use of VAN for gram-positive coverage in empiric antimicrobial therapy of neutropenic patients (Fig. 4).

The outbreak was contained as of June 2014, however until the end of 2017 sixteen new cases were detected at hospital 4 at a rate of one per 2.6 months (Fig. 1). The fact that nearly half of these later cases were LREfm clones other than the outbreak clone could be revealed by WGS only retrospectively.

\section{Discussion}

In this study, we report the first LREfm outbreak in an Austrian hospital, which involved colonized as well as infected patients. WGS identified a hospital-adapted ST117, CT24 LREfm clone as the main causative agent of the outbreak in the affected hemato-oncology department. There was high clonality among this cluster, since all strains but one were ST117, CT24, and differed by 


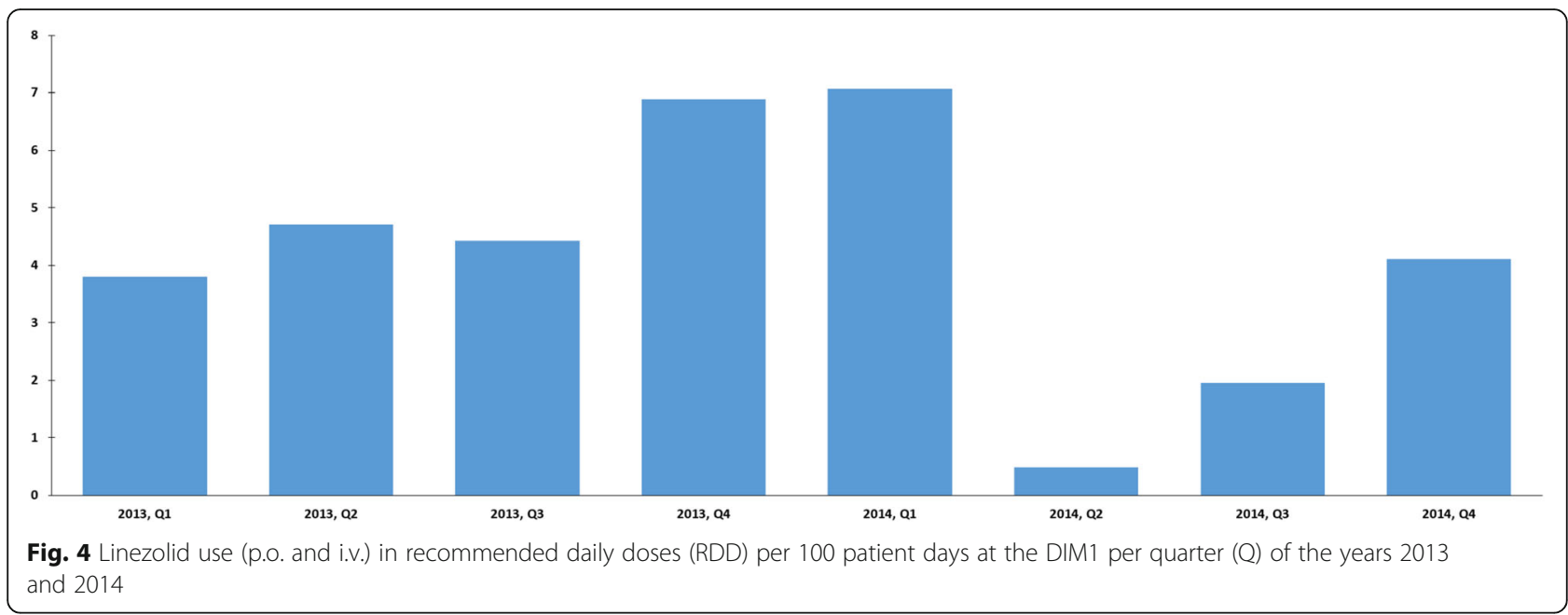

$\leq 10$ alleles. Interestingly, the outbreak clone did not spread to any other departments within hospital 4, meaning that the source was confined to DIM1. E. faecium ST117 is a widely disseminated clone that may present resistance to many drugs, including LZD [25]. In particular, ST117, CT24 has been previously identified in outbreaks caused by VAN-resistant E. faecium [26, 27] although up to our knowledge, this clone has been never associated to LREfm outbreaks.

The addition of isolates from patient collectives B and $\mathrm{C}$ allowed us to gain more insight into the distribution of $E$. faecium between different healthcare facilities, especially for the region of Upper Austria. WGS also helped to elucidate that the outbreak at hospital 4 not just consisted of the cluster 1 clone (ST117, CT24), but also of two other clusters (2 and 3 ) and multiple unclustered isolates, suggesting several separate introductions of LREfm.

Cluster 2 (ST80, CT1873) indicates a continuous spillover between four healthcare facilities located in the same province even though patient movement could not be analyzed comprehensively. Cluster 3 (ST80, CT16) represented a separated cluster within the DIM1, in which a patient showed loss of LREfm during a one year period. Similarly, in cluster 4 (ST80, CT315) we could observe transmission of E. faecium between hospitals 4 and 6, although the isolate detected at hospital 6 was LSEfm, which might indicate the loss of the resistance trait in this strain. Concerning cluster 6 (ST117, CT929), available epidemiological data do not allow to understand why two isolates from different time periods and geographical areas clustered together.

The clustering together of LREfm and LSEfm in nearly all the clusters is an interesting finding in our study and, to our knowledge, it has not been described previously. However, outbreak reports usually only include resistant isolates [28]. An explanation for our finding of genetically closely related LREfm and LSEfm isolates may be the different antibiotic selective pressure in diverse hospitals.

All LREfm cases received concurrent antibiotic treatment, which may account for selection of enterococci in the enteric microbiome. Recent exposure to LZD was found only in a minority $(n=10 ; 27.8 \%)$ of our cases, however, highlighting the contribution of environmental and person-to-person spread as previously described [25]. This is supported by the fact that for the majority of DIM1 cases close physical contact could be confirmed. Hemato-oncological patients generally are at high risk for infections, but with profound immunosuppression, frequent hospitalization and repeated administration of antibiotics they are especially prone to acquisition of multidrug-resistant E. faecium [29]. The association of acquisition of the outbreak strain with screening and death during follow-up, however, is most likely confounded by the intrinsic prognosis and type of care of this patient population.

All LREfm isolates were clearly LZD resistant by disk diffusion testing. MIC testing yielded a wide range of concentrations but those were not associated with the number of mutated 23S rRNA genes, in contrast to previous reports $[4,5]$. TZD MICs have been previously shown to be lower than LZD MICs in E. feacium, which was also the case in our study [30]. However, the clinical significance of this is unknown, since clinical breakpoints have not yet been defined.

Interestingly, a genetic basis for the phenotypic LZD resistance could not be identified in all isolates. The only resistance mechanism identified in 14 (31.1\%) of the LREfm in our study was the G2576U point mutation in the $23 \mathrm{~S}$ rRNA, meaning that additional resistance mechanisms must exist among Austrian LREfm isolates, as proposed elsewhere [31]. According to a recent systematic review [32], up to 80.5\% of LREfm carry the point mutation G2576U, 
although other authors have reported lower frequencies [10]. The previously undescribed additional point mutation A2598G is of unknown significance, however it was found only in three LREfm and only in combination with the G2576U mutation. The plasmid-mediated LZD resistance genes optrA, cfr and poxtA could not be detected by PCR nor by WGS.

Regarding resistance mechanisms apart from ribosomal point mutations, in a recent study on Mycobacterium abscessus [33], authors identified efflux pumps $\operatorname{lm} r S$ and $m m p L 9$ at higher transcriptional levels among LZD-resistant isolates, suggesting an association with the resistant phenotype. Similarly, one could hypothesize that efflux pumps may contribute to LZD resistance, since efmA was detected among nearly all LREfm isolates. In addition, $d f r \mathrm{~F}$, which encodes for a chromosomal dihydrofolate reductase [34], was significantly associated (Fisher's exact $=0.002$ ) with the LREfm phenotype. Moreover, from a total of 11 cases that were treated with trimethoprim/sulfamethoxazole, eight cases belonged to the outbreak cluster. Trimethoprim is known to act against the reductase and sulfonamides have been described as a risk factor for LZD resistance acquisition [11].

Concerning the VGs, most of them (7/10) have been associated with more pathogenic E. faecium clones [25]. In accordance to other studies, $\operatorname{sgr} A, a c m, e c b A$ are usually present at high frequencies in E. faecium strains of clinical origin $[35,36]$. We did find an association between the adherence gene ecbA and the LREfm phenotype. Similarly, $e c b \mathrm{~A}$ has been found in E. faecium outbreaks [37].

In our setting, the established control measures managed to terminate the initial outbreak event, although they did not prevent later sporadic cases. Unfortunately, environmental sampling was not performed and therefore an environmental reservoir was not identified. Nevertheless, the persistence of enterococci, especially of ST117, in hospital settings is well known [38] and as it has been shown here, high-risk populations such as hemato-oncological patients account for most of the cases. Fortunately, most patients were only temporarily colonized with LREfm and only a minority of them developed infection.

A limitation of our study was the lack of systematic testing for clearance of LREfm carriage, although our data suggest that in most patients LREfm do not seem to become established for a longer time period. Nevertheless, it is important to promptly detect even LREfm colonization since such patients may be a reservoir of transmission to patients of the same ward, the same hospital or even within a healthcare network. Also, colonization generally precedes invasive infections which may be difficult to treat, especially when other resistance mechanisms are present.

\section{Conclusions}

We have described an outbreak caused by Linezolid resistant Enterococcus faecium (LREfm) in an Austrian hematooncology unit. Whole-genome sequencing was a useful method to investigate isolates from the outbreak hospital and to compare them with strains from other healthcare facilities in Austria. The outbreak-causing strain was identified as highly clonal hospital adapted ST117, CT24 LREfm. Multiple additional clusters were identified, partly explaining the perceived repeated occurrence of LREfm after termination of the outbreak by multi-modal infection control measures. In a high-risk setting, active surveillance of LREfm is important for prompt outbreak detection.

\section{Additional file}

Additional file 1: Table S1. Resistance genes and point mutations at the $23 \mathrm{~S}$ rRNA found among the sequenced strains. Table S2. Virulence genes carried by the 54 sequenced strains and resistance phenotype. (XLSX $17 \mathrm{~kb}$ )

\section{Abbreviations}

CC: clonal complex; cgMLST: Core genome multi locus sequence typing; CT: cluster type; DIM1: Department of Internal Medicine 1; EUCAST: European Committee on Antimicrobial Susceptibility Testing; LREfm: Linezolid resistant Enterococcus faecium; LSEfm: Linezolid susceptible Enterococcus faecium; LZD: Linezolid; ST: Sequence type; VAN: Vancomycin; VRE: Vancomycin resistant enterococci; WGS: Whole genome sequencing

\section{Acknowledgements \\ The authors thank Anna Blaimschein, Bernadette Hones, Sarah Widhalm and Anna Stöger for excellent technical work, Sieglinde Sorschag for providing clinical data, Marianne Graninger for assisting with data collection, and Claudia Kastner for her help to obtain linezolid usage data. Tedizolid gradient strips were generously donated by Merck Sharp \& Dome, Vienna.}

\section{Authors' contributions}

HK and AC contributed equally and share first authorship. Study design: HK, AC. Epidemiological study: HK, AC, SMS. Microbiological analysis: HK, RH, PA. WGS: AC, WR. Data analysis: HK, AC, WR. Manuscript writing: HK, AC. Manuscript revision: FA, PA, WR. All authors read and approved the final manuscript.

\section{Funding}

This work was supported by a grant from the European Public Health Microbiology Training Programme (EUPHEM), European Centre for Disease Prevention and Control (specific grant agreement number 1 ECD.7550 implementing ECDC/GRANT/2017/003) to Adriana Cabal Rosel.

\section{Availability of data and materials}

The raw WGS reads were deposited into Sequence Read Archive (SRA) database under NCBI accession PRJNA541232. The datasets used and/or analyzed during the current study are either included in the published article and its supplementary files or available from the corresponding author on reasonable request.

\section{Ethics approval and consent to participate}

The study was approved by the local ethics committee (vote ECS 1053/ 2018)

\section{Consent for publication}

Not applicable.

Competing interests

The authors declare that they have no competing interests. 


\section{Author details}

National Reference Center for Antimicrobial Resistance and Nosocomial Infections, Institute for Hygiene, Microbiology and Tropical Medicine, Ordensklinikum Linz Elisabethinen, Fadingerstrasse 1, 4020 Linz, Austria. ${ }^{2}$ AGES - Austrian Agency for Health and Food Safety, Institute of Medical Microbiology and Hygiene, Waehringerstrasse 25a, 1090 Vienna, Austria. ${ }^{3}$ European Public Health Microbiology Training Programme (EUPHEM), European Centre for Disease Prevention and Control (ECDC), Stockholm, Sweden. ${ }^{4}$ Department of Internal Medicine 1, Ordensklinikum Linz Elisabethinen, Fadingerstrasse 1, 4020 Linz, Austria.

\section{Received: 30 May 2019 Accepted: 15 August 2019}

\section{Published online: 09 September 2019}

\section{References}

1. Dubin K, Pamer EG. Enterococci and their interactions with the intestinal microbiome. Microbiol Spectr. 2014,5:10.

2. Gao W, Howden BP, Stinear TP. Evolution of virulence in Enterococcus faecium, a hospital-adapted opportunistic pathogen. Curr Opin Microbiol. 2018:41:76-82

3. Lee T, Pang S, Abraham S, Coombs GW. Antimicrobial-resistant CC17 Enterococcus faecium: the past, the present and the future. J Glob Antimicrob Resist. 2019;16:36-47.

4. Cattoir V, Giard J-C. Antibiotic resistance in Enterococcus faecium clinica isolates. Expert Rev Anti-Infect Ther. 2014;12:239-48.

5. Marshall SH, Donskey CJ, Hutton-Thomas R, Salata RA, Rice LB. Gene dosage and linezolid resistance in Enterococcus faecium and Enterococcus faecalis. Antimicrob Agents Chemother. 2002;46:3334-6.

6. Bourgeois-Nicolaos N, Massias L, Couson B, Butel M-J, Andremont A Doucet-Populaire F. Dose dependence of emergence of resistance to linezolid in Enterococcus faecalis in vivo. J Infect Dis. 2007:195:1480-8.

7. Diaz L, Kiratisin P, Mendes RE, Panesso D, Singh KV, Arias CA. Transferable plasmid-mediated resistance to linezolid due to cfr in a human clinical isolate of Enterococcus faecalis. Antimicrob Agents Chemother. 2012;56:3917-22.

8. Wang Y, LV Y, Cai J, Schwarz S, Cui L, Hu Z, et al. A novel gene, optrA, that confers transferable resistance to oxazolidinones and phenicols and its presence in Enterococcus faecalis and Enterococcus faecium of human and animal origin. J Antimicrob Chemother. 2015;70:2182-90.

9. Antonelli A, D'Andrea MM, Brenciani A, Galeotti CL, Morroni G, Pollini S, et al. Characterization of poxtA, a novel phenicol-oxazolidinone-tetracycline resistance gene from an MRSA of clinical origin. J Antimicrob Chemother. 2018:73:1763-9.

10. Lee SM, Huh HJ, Song DJ, Shim HJ, Park KS, Kang Cl, et al. Resistance mechanisms of linezolid-nonsusceptible enterococci in Korea: low rate of $23 \mathrm{~S}$ rRNA mutations in Enterococcus faecium. J Med Microbiol. 2017;66:1730-5

11. McGregor JC, Hartung DM, Allen GP, Taplitz RA, Traver R, Tong T, et al. Risk factors associated with linezolid-nonsusceptible enterococcal infections. Am J Infect Control. 2012:40:886-7.

12. Greene MH, Harris BD, Nesbitt WJ, Watson ML, Wright PW, Talbot TR, et al. Risk Factors and Outcomes Associated With Acquisition of Daptomycin and Linezolid-Nonsusceptible Vancomycin-Resistant Enterococcus. Open Forum Infect Dis. 2018;5:ofy185.

13. Niebel M, Perera MTPR, Shah T, Marudanayagam R, Martin K, Oppenheim $B A$, et al. Emergence of linezolid resistance in hepatobiliary infections caused by Enterococcus faecium. Liver Transpl. 2016:22:201-8.

14. Ntokou E, Stathopoulos C, Kristo I, Dimitroulia E, Labrou M, Vasdeki A, et al Intensive care unit dissemination of multiple clones of linezolid-resistant Enterococcus faecalis and Enterococcus faecium. J Antimicrob Chemother 2012:67:1819-23.

15. Robert-Koch-Institut. Antibiotika-Verbrauchs-Surveillance (Available from: https://avs.rki.de/)

16. Brenciani A, Morroni G, Vincenzi C, Manso E, Mingoia M, Giovanetti E, et al Detection in Italy of two clinical Enterococcus faecium isolates carrying both the oxazolidinone and phenicol resistance gene optrA and a silent multiresistance gene cfr. J Antimicrob Chemother. 2016;71:1118-9.

17. Nurk S, Bankevich A, Antipov D, Gurevich AA, Korobeynikov A, Lapidus A, et al. Assembling single-cell genomes and mini-metagenomes from chimeric MDA products. J Comput Biol. 2013;20:714-37.

18. de Been M, Pinholt M, Top J, Bletz S, Mellmann A, van Schaik W, et al. Core genome multilocus sequence typing scheme for high- resolution typing of Enterococcus faecium. J Clin Microbiol. 2015;53:3788-97.
19. Jolley KA, Maiden MC. Using multilocus sequence typing to study bacterial variation: prospects in the genomic era. Future Microbiol. 2014:9:623-30.

20. Jia B, Raphenya AR, Alcock B, Waglechner N, Guo P, Tsang KK, et al. CARD 2017: expansion and model-centric curation of the comprehensive antibiotic resistance database. Nucleic Acids Res. 2017;45:D566-d73.

21. Hasman H, Clausen P, Kaya H, Hansen F, Knudsen JD, Wang M, et al. LREfinder, a web tool for detection of the 23S rRNA mutations and the optrA $\mathrm{cfr}, \mathrm{cfr}(\mathrm{B})$ and poxtA genes encoding linezolid resistance in enterococci from whole-genome sequences. J Antimicrob Chemother. 2019;74:1473-6.

22. Joensen KG, Scheutz F, Lund O, Hasman H, Kaas RS, Nielsen EM, et al. Realtime whole-genome sequencing for routine typing, surveillance, and outbreak detection of verotoxigenic Escherichia coli. J Clin Microbiol. 2014; $52: 1501-10$

23. Liu B, Zheng D, Jin Q, Chen L, Yang J. VFDB 2019: a comparative pathogenomic platform with an interactive web interface. Nucleic Acids Res. 2019;47:D687-d92.

24. Carattoli A, Zankari E, Garcia-Fernandez A, Voldby Larsen M, Lund O, Villa L, et al. In silico detection and typing of plasmids using PlasmidFinder and plasmid multilocus sequence typing. Antimicrob Agents Chemother. 2014; 58:3895-903.

25. Hegstad K, Longva JA, Hide R, Aasnaes B, Lunde TM, Simonsen GS. Cluster of linezolid-resistant Enterococcus faecium ST117 in Norwegian hospitals. Scan J Infect Dis. 2014;46:712-5.

26. Hammerum AM, Baig S, Kamel Y, Roer L, Pinholt M, Gumpert H, et al. Emergence of vanA Enterococcus faecium in Denmark, 2005-15. J Antimicrob Chemother. 2017;72:2184-90.

27. Zhou X, Chlebowicz MA, Bathoorn E, Rosema S, Couto N, Lokate M, et al. Elucidating vancomycin-resistant Enterococcus faecium outbreaks: the role of clonal spread and movement of mobile genetic elements. J Antimicrob Chemother. 2018;73:3259-67.

28. Dobbs TE, Patel M, Waites KB, Moser SA, Stamm AM, Hoesley CJ. Nosocomial spread of Enterococcus faecium resistant to vancomycin and linezolid in a tertiary care medical center. J Clin Microbiol. 2006:44:3368-70.

29. Hayakawa K, Marchaim D, Pogue JM, Ho K, Parveen S, Nanjireddy P, et al. Predictors and outcomes of linezolid-resistant vancomycin-resistant Enterococcus: a case-case-control study. Am J Infect Control. 2012;40:e261-3.

30. Chen $\mathrm{K}-\mathrm{H}$, Huang $\mathrm{Y}-\mathrm{T}$, Liao $\mathrm{C}-\mathrm{H}$, Sheng $\mathrm{W}-\mathrm{H}$, Hsueh $\mathrm{P}-\mathrm{R}$. In vitro activities of Tedizolid and linezolid against gram-positive cocci associated with acute bacterial skin and skin structure infections and pneumonia. Antimicrob Agents Chemother. 2015;59:6262-5.

31. Klupp EM, Both A, Belmar Campos C, Buttner H, Konig C, Christopeit M, et al. Tedizolid susceptibility in linezolid- and vancomycin-resistant Enterococcus faecium isolates. Eur J Clin Microbiol Infect Dis. 2016;35:1957-61.

32. Bi R, Qin T, Fan W, Ma P, Gu B. The emerging problem of linezolid-resistant enterococci. J Glob Antimicrob Resist. 2018;13:11-9.

33. Ye M, Xu L, Zou Y, Li B, Guo Q, Zhang Y, et al. Molecular analysis of linezolid-resistant clinical isolates of Mycobacterium abscessus. Antimicrob Agents Chemother. 2019:63:e01842-18.

34. Bergmann R, van der Linden M, Chhatwal GS, Nitsche-Schmitz DP. Factors that cause trimethoprim resistance in streptococcus pyogenes. Antimicrob Agents Chemother. 2014;58:2281-8.

35. Freitas AR, Tedim AP, Novais C, Coque TM, Peixe L. Distribution of putative virulence markers in Enterococcus faecium: towards a safety profile review. J Antimicrob Chemother. 2017;73:306-19.

36. Arshadi M, Mahmoudi M, Motahar MS, Soltani S, Pourmand MR. Virulence determinants and antimicrobial resistance patterns of vancomycin-resistant Enterococcus faecium isolated from different sources in Southwest Iran. Iran J Public Health. 2018;47:264-72.

37. Hendrickx APA, van Luit-Asbroek M, Schapendonk CME, van Wamel WJB, Braat JC, Wijnands LM, et al. SgrA, a Nidogen-binding LPXTG surface Adhesin implicated in biofilm formation, and EcbA, a collagen binding MSCRAMM, are two novel Adhesins of hospital-acquired emEnterococcus faecium. Infect Immun. 2009;77:5097-106.

38. Sanchez-Diaz AM, Cuartero C, Lozano S, Rodriguez JD, Alonso JM, QuilesMelero I, et al. Emergence and long-lasting persistence of linezolid-resistant Enterococcus faecium-ST117 in an oncohematologic patient after a nine-day course of linezolid. Microbial drug resistance (Larchmont, NY). 2014;20:17-21.

\section{Publisher's Note}

Springer Nature remains neutral with regard to jurisdictional claims in published maps and institutional affiliations. 